\title{
The Impact of E-Games On Adolescents' Social Development
}

\author{
Kostaki, Dimitra and Ganetsou, Evanthia \\ The American College of Greece - Deree College, Greece
}

\begin{abstract}
Multiplayer video games receive increasing attention, not only within the gaming community and IT members, but also among psychology scholars, as they have been found to enhance adolescents' social dexterities. The social experience of e-gaming promotes extraversion, as social hobby platforms furnish a pretext where interdependency among gamers is crucial for success in the game (e.g. Star Wars Galaxy). E-games further serve as cooperative and collaborative contexts where team-oriented play and joined cooperative scripts are primed to establish a high ranking. Gamers work closely towards searching for enemies, sharing strategies, equipment, and mindset, and coordinating plans to eliminate threats (e.g. Call of Duty). Effective interpersonal communication also increases the chances of winning. During warfare, adolescents allocate a substantial amount of time communicating with fellow players; conversations within a game include fast, short replies on partner's names, object or opponent locations, and problem-solving strategies (e.g. World of Warcraft). Moreover, e-games enhance prosocial behaviors among adolescents by providing a context where supportive practices, empathy, altruism, and encouragement are practiced (e.g. Super Mario Sunshine). Helping behaviors are reinforced even during violent e-games and often generalize to friends and family outside the gaming community. Video games are virtual spaces that allow gamers from all around the world to develop meaningful friendships and romantic relationships, or to strengthen pre-existing friendship ties. Interactions among gamers offer a sense of acceptance and belonging in a wider social gaming community, where players learn to trust one another, value each other's advice, and share their experiences (e.g. Among Us).
\end{abstract}

Keywords: multiplayer video games; social skills; developmental psychology 